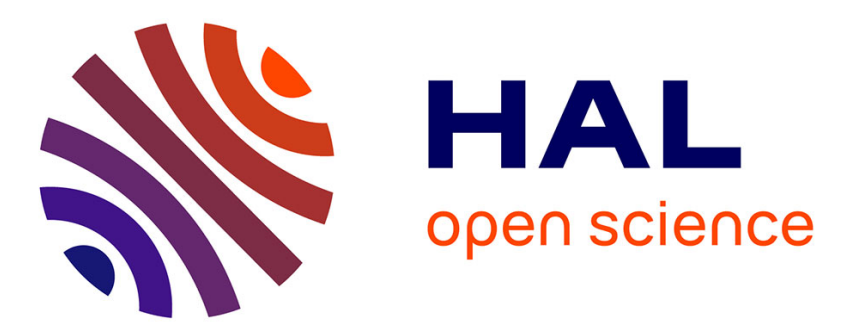

\title{
Symbolic simultaneous registration and change detection between two detection sets in the mine warfare context
}

Nicolas Florian, Andreas Arnold Bos, Isabelle Quidu, Benoit Zerr

\section{To cite this version:}

Nicolas Florian, Andreas Arnold Bos, Isabelle Quidu, Benoit Zerr. Symbolic simultaneous registration and change detection between two detection sets in the mine warfare context. IEEE Oceans 2019, Jun 2019, Marseille, France. 10.1109/OCEANSE.2019.8867211 . hal-02186401

\section{HAL Id: hal-02186401 \\ https://hal-ensta-bretagne.archives-ouvertes.fr/hal-02186401}

Submitted on 17 Jul 2019

HAL is a multi-disciplinary open access archive for the deposit and dissemination of scientific research documents, whether they are published or not. The documents may come from teaching and research institutions in France or abroad, or from public or private research centers.
L'archive ouverte pluridisciplinaire HAL, est destinée au dépôt et à la diffusion de documents scientifiques de niveau recherche, publiés ou non, émanant des établissements d'enseignement et de recherche français ou étrangers, des laboratoires publics ou privés. 


\title{
Symbolic Simultaneous Registration and Change Detection Between Two Detection Sets In the Mine Warfare Context
}

\author{
Florian Nicolas*†, Andreas Arnold-Bos*, Isabelle Quidu ${ }^{\dagger}$, Benoit Zerr ${ }^{\dagger}$ \\ ${ }^{*}$ THALES DMS FRANCE S.A.S, Brest, France \\ \{florian.nicolas, andreas.arnold-bos\}@ fr.thalesgroup.com \\ ${ }^{\dagger}$ Lab-STICC, UMR CNRS 6285, ENSTA Bretagne, Brest, France \\ \{florian.nicolas, isabelle.quidu, benoit.zerr\}@ensta-bretagne.org
}

\begin{abstract}
In the underwater mine warfare context, change detection is a principle consisting in comparing a newly sensed seabed area, usually by means of a side scan sonar, to another one that has potentially been sensed several months or years ago. In this paper, we propose an approach to simultaneously register (i.e geometrically align) the reference and the repeated data while detecting new and missing objects between both datasets acquisition. This method is first evaluated on data provided by a simulator based on a model of navigation uncertainty as well as on error sources due to the imaging sonar, in order to assess its robustness against different parameters. We also provide results on datasets acquired at sea and demonstrate its efficiency to solve the change detection problem.

Index Terms-Image registration, change detection, synthetic aperture sonar, iterative closest point, mine warfare, side scan sonar
\end{abstract}

\section{INTRODUCTION}

Underwater Mine Warfare operations usually consist, by means of sonars, in a succession of steps namely the detection, localization, classification, identification and potentially the destruction of underwater threats such as manufactured mines or improvised explosive devices (IED).

While the previously introduced steps are the right procedure to follow in case of a newly surveyed area, another approach can be thought of when the surveyed area has already been sensed in the past. Such an approach, known as change detection (CD), aims at comparing such previously acquired data, said as reference data, with newly sensed data, the repeated ones, in order to detect potential changes arisen between both acquisitions.

However, the reference and repeated images cannot be directly compared. Indeed, due to errors from various sources during the data acquisition, when projected into the Earth frame, identical features from both datasets will not match (i.e they will not be geometrically aligned). Thus, a first step, data registration, which will geometrically align both datasets, must generally be the very first step of a change detection processing chain.

In the sonar imaging community, registration methods and more widely, change detection ones, are usually split into two groups, namely the image-based (or iconic) and objectbased (or symbolic) ones. While the former directly work on raw pixel intensities to compute the optimal geometric transformation [1]-[5] and detect changes [6], [7], the latter rather independently detect features, such as, for example, mine-like objects (MILCOs) or homogeneous seabed areas, within both the reference and repeated tracks before matching them. Thus, such an optimal transformation can be computed [8]-[11] and the said change detection step performed [12].

Among the image-based methods, we distinguish between coherent methods [13]-[18], relying on complex signals, and incoherent ones, taking as input only the amplitude of the backscattered signals [6], [16]. However, while coherent methods are able to detect tiny changes compared to incoherent ones, they have several drawbacks. Indeed, first, they need a highly accurate registration step such as one tenth of a pixel as stated in [18] to achieve sufficient coherence between the reference and repeated images. Secondly, the time delay between both tracks sensing must be short enough, from few hours to few days, to ensure this coherence to be high enough [19] to allow the use of such coherent methods. As, in our context, several months can elapse between the acquisition of the reference and the repeated data, we cannot consider such methods.

In this paper, we thus propose a framework allowing to simultaneously perform the registration and change detection steps from a symbolic perspective. Such an object-based approach has been chosen in order to overcome limitations of image-based methods, such as aspect or grazing angles variability [6], which might potentially yield a high false alarms rate regarding change detection performances. Indeed, in the symbolic case, such variabilities can be handled within the features extractor, namely an automatic target recognition (ATR), system which works on raw sonar data (i.e. into the sensor frame). 


\section{UNDERWATER NAVIGATION AND FEATURES POSITIONING}

\section{A. Underwater navigation devices}

As GPS signals are not available underwater, the estimation of the underwater location of a moving carrier must rely on other sensors such as inertial navigation systems (INS), Doppler velocity logs (DVL) or acoustic positioning systems, just to name a few. Data from such sensors can be fused by means of state estimators such as, for example, a Kalman filter [20]. Simultaneous localization and mapping (SLAM), also known as concurrent mapping and localization (CML)[21], is another alternative to underwater navigation. SLAM techniques consist in performing observations (from sensors equipping the carrier, such as imaging or ranging sonar [22], [23]), in order to extract features allowing to build a map, while keeping track of its position by re-observing such features to estimate its location within the map.

\section{B. DVL-aided INS uncertainty navigation model}

In this paper, we focus on an autonomous underwater vehicle (AUV) equipped with an INS, a DVL, a synthetic aperture sonar (SAS) sensor and also on a GPS sensor to allow for surface navigation.

We thus hereafter aim at providing the full navigation model for such a system.

The trajectory of an AUV relying on a DVL-aided INS for its navigation will geometrically drift from the expected one, and such a geometrical transformation can be described by [24]:

- a scale factor $s$ proportional to the speed $\left(28.8 \mathrm{~m} \cdot \mathrm{h}^{-1}\right.$ for a speed of $2 \mathrm{~m} . \mathrm{s}^{-1}$ mentioned in [25]). This term can come from a sound speed estimation error, the echo's direction of arrival variation because of the carrier motion or a transducers misalignment during the DVL installation on the carrier.

- a rotational term, $\Psi$, also due to a misalignment between the DVL and/or the carrier or/and the INS, during the mechanical installation.

An illustration of this long term accuracy error, denoted by $\epsilon_{\mathrm{LTA}}$, is provided in (Fig.1) and can mathematically be formulated as,

$$
\begin{aligned}
\epsilon_{\mathrm{LTA}} & =D(t) \sqrt{1+s^{2}-2 s \cos \Psi} \\
& =K_{\mathrm{LTA}} \times D(t),
\end{aligned}
$$

where $D(t)$ is the true (but unobserved) distance, as the crow flies, from the last GPS measurement (i.e. before the carrier's diving) and the current location of the carrier.

Additionally, each pulse emitted by the DVL, with a repetition frequency $\frac{1}{\Delta T}$, is also polluted by an error denoted as $\epsilon_{\mathrm{STA}, \text { single }}$ (2)[26] and depending on the carrier's speed $V$. Thus, the integration of such pulses on a duration $\Delta T$, will sum up such errors yielding what is often referred to as a random walk [27] or a brownian motion process (3).

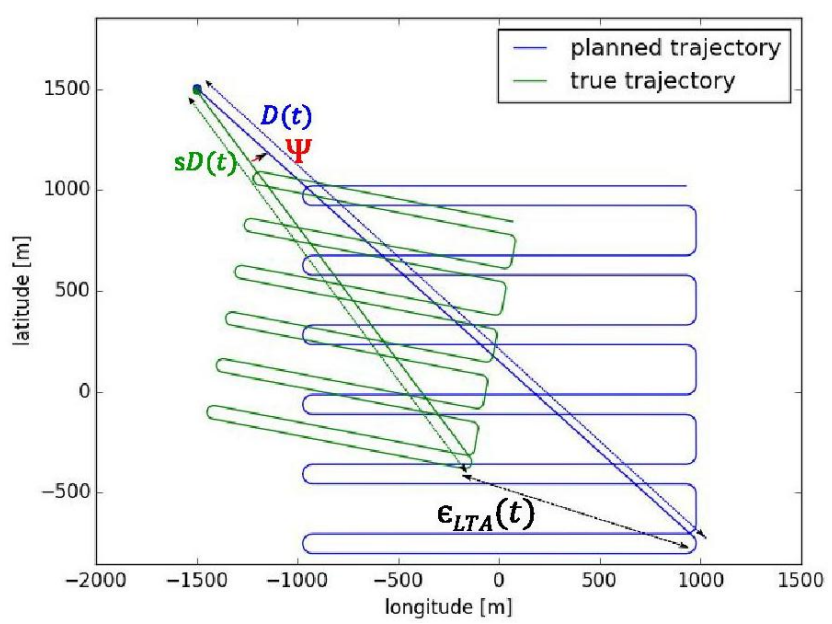

Fig. 1: Planned trajectory versus true (unobserved) trajectory. The misalignment and scale factor are highly exagerated in this picture. $D(t)$ : distance from last GPS measurement to current location as the crow flies; $s$ : scale factor; $\Psi$ : misalignment angle; $\epsilon_{\mathrm{LTA}}$ : error.

$$
\begin{aligned}
\epsilon_{\mathrm{STA}, \text { single }} & \sim \mathcal{N}\left(0, \sigma_{\mathrm{STA}, \text { single }}^{2}\right) \\
\sigma_{\mathrm{STA}, \text { single }} & =K_{\mathrm{STA}} \times \sqrt{V}
\end{aligned}
$$

A value of $2.33 \times 10^{-1}$ for $K_{\text {STA }}$ is presented in [25].

$$
\begin{aligned}
\sigma_{S T A}^{2}(t) & =\frac{1}{\Delta T} \int_{0}^{t}\left(\sigma_{\mathrm{STA}, \text { single }} \times \Delta T\right)^{2} d \tau \\
& =\sigma_{\text {STA, single }}^{2} \times \Delta T \times t \\
& =K_{\text {STA }}^{2} \times V \times \Delta T \times t
\end{aligned}
$$

Before diving, the AUV location is computed by means of GPS measurements. Considering our change detection problem, where two datasets have to be compared, we consider the GPS measurements from both missions to be independent. Thus, while, at short term, such measurements are correlated [28], we assume the elapsed time between both datasets acquisition to be significant enough ( $i \dot{i} 1$ hour) to make such an assumption.

This GPS bias, whose standard deviation will depend on the considered equipment (DGPS, Precise Point Positioning, ...), is modeled as a Rayleigh distribution, with parameter $\sigma_{\mathrm{GPS}}$ and standard deviation $r_{\mathrm{GPS}}$ related by

$$
\sigma_{\mathrm{GPS}}=r_{\mathrm{GPS}} \sqrt{\frac{2}{4-\pi}} .
$$

Both the latitude and longitude components, under an independent assumption, can thus be modeled as

$$
t_{\mathrm{GPS}}^{(.)} \sim \mathcal{N}\left(0, \sigma_{\mathrm{GPS}}^{2}\right) .
$$

The navigation error, in an Earth-centered frame, can thus be written 


$$
\boldsymbol{\Sigma}_{\text {nav }}(t)=\left[\sigma_{\text {GPS }}^{2}+\sigma_{\text {STA }}^{2}(\sqrt{t})+\sigma_{\text {LTA }}^{2}(D(t))\right] \mathbf{I}_{\mathbf{2}}
$$

\section{Sonar-related position errors}

So far, we have only considered error sources due to the full navigation system. However, in the mine-warfare context, where we aim at locating objects laid on seabed, the side-scan sonar itself can also be an error source. Such errors can mainly come from sound speed estimation, bearing measurement and altitude estimation. In the far field assumption we deal with, sound speed and bearing errors prevail, and they are thus modeled within our simulator.

1) Uncertainty on sound speed estimation: If we consider a celerity error $\Delta c \sim \mathcal{N}\left(0, \sigma_{c}^{2}\right)$. From (Fig.2) we can write,

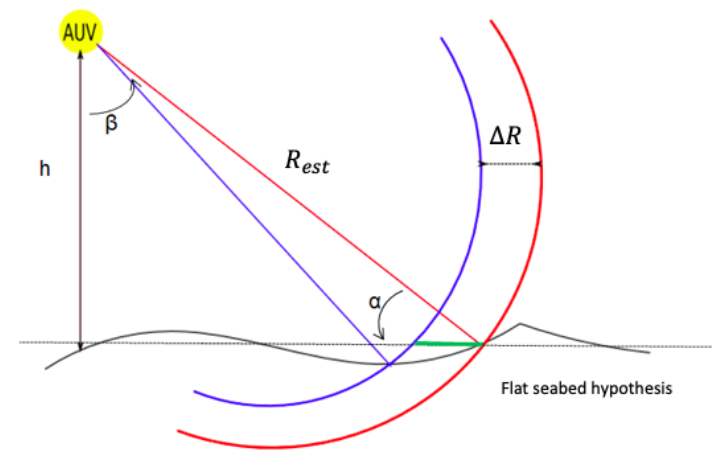

Fig. 2: Effect of a misalignment error on an object position estimation

$$
\begin{aligned}
R_{\text {est }} & =\frac{\left(c_{0}+\Delta c\right) \Delta t}{2} \\
R_{\text {true }} & =\frac{c_{0} \Delta t}{2}
\end{aligned},
$$

where $\Delta t$ is the two-way time delay of the acoustic wave, thus

$$
\Delta R=\frac{\Delta c \Delta t}{2}
$$

that we can write

$$
\Delta R=\frac{\Delta c R_{\mathrm{est}}}{c_{0}+\Delta c} \stackrel{\Delta c \ll c_{0}}{\approx} \frac{\Delta c R_{\mathrm{est}}}{c_{0}},
$$

The grazing angle $\alpha$ is

$$
\alpha=\arcsin \left(\frac{h}{R_{\mathrm{est}}}\right) .
$$

Under a flat seabed assumption, we have

$$
\begin{aligned}
\Delta d_{\text {seabed }} & =\cos (\alpha) \Delta R \\
& =\cos \left(\arcsin \left(\frac{h}{R_{\text {est }}}\right)\right) \Delta R \\
& =\sqrt{1-\left(\frac{h}{R_{\text {est }}}\right)^{2}} \Delta R
\end{aligned}
$$

The variance on the across-track axis, in the AUV-centered frame, in terms of ground distance, can thus be written as

$$
\sigma_{\mathrm{accros}}^{2}=\left(1-\left(\frac{h}{R_{\mathrm{est}}}\right)^{2}\right) \frac{\sigma_{c}^{2} R_{\mathrm{est}}^{2}}{c_{0}^{2}} .
$$

2) Antenna misalignment: If we now consider, still in the AUV-centered frame, an angular misalignment of the antenna (Fig.3) described as $\theta_{\text {mis }} \sim \mathcal{N}\left(0, \sigma_{\text {mis }}^{2}\right)$, we can write

$$
\Delta d_{\text {along }}=R_{\text {est }} \sin \left(\theta_{\text {mis }}\right) \stackrel{\theta_{\text {mis }} \ll 1}{\approx} R_{\text {est }} \theta_{\text {mis }},
$$

thus

$$
\sigma_{\text {along }}^{2}=R_{\text {est }}^{2} \sigma_{\text {mis }}^{2} .
$$

The covariance matrix is thus

$$
\boldsymbol{\Sigma}_{\mathrm{SSS}}=\left(\begin{array}{cc}
R_{\mathrm{est}}^{2} \sigma_{\mathrm{mis}}^{2} & 0 \\
0 & \left(1-\left(\frac{h}{R_{\mathrm{est}}}\right)^{2}\right) \frac{\sigma_{c}^{2} R_{\text {est }}^{2}}{c_{0}^{\text {es }}}
\end{array}\right)
$$

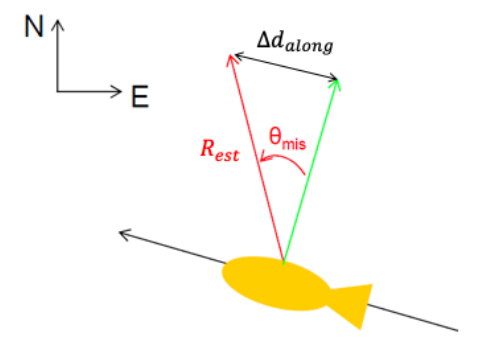

Fig. 3: Effect of a misalignment error on an object position estimation

Finally, the uncertainty model of the location of any sensed object of interest laid on the seafloor is, in an Earth-centered frame

$$
\boldsymbol{\Sigma}_{\mathrm{loc}}=\boldsymbol{\Sigma}_{\mathrm{nav}}+\boldsymbol{R}_{\mathrm{AUV} \dashv \text { Earth }} \boldsymbol{\Sigma}_{\mathrm{SSS}} \boldsymbol{R}_{\mathrm{AUV} \dashv \text { Earth }}^{\mathrm{T}},
$$

where $\boldsymbol{R}_{\mathrm{AUV} \rightarrow \text { Earth }}$ is the change of frame matrix from the AUV centered frame to the Earth-centered one.

Nonetheless, we emphasize that such a model is only an approximation as it does not take into account all the potential error sources. Indeed, the carrier's altitude has been assumed to be exactly measured while the seafloor has also been supposed to be flat. Another simplification comes from the estimated position of a mine-like objects. Indeed, such objects, classically detected by means of an automatic target detection/recognition algorithm (ATD/R) [29]-[32], are not isolated points and their estimated positions can thus depend on the grazing or view angles under which these objects are sensed. 


\section{Design of a symbolic simulator}

In order to assess the performances of a symbolic registration and/or change detection algorithm, we felt the need to design a simulator able to generate inputs to such algorithms. Indeed, as the change detection issue is strongly imbalanced (i.e. the absence of change is much more regular than real ones), relying only on data acquired at sea makes such an assessment difficult. The simulator functioning, mainly based on the previously introduced navigation model, is detailed in (Alg.1), yields two pointsets, by means of two trajectories, namely the reference and repeated ones which are respectively denoted by $\mathcal{C}^{(r e f)}$ and $\mathcal{C}^{(r e p)}$. The repeated detection set is computed after removing a rate of $r_{\text {dis }}$ existing objects, to simulate objects disappearance, and adding a rate of $r_{\text {new }}$ new objects, to simulate objects appearance.

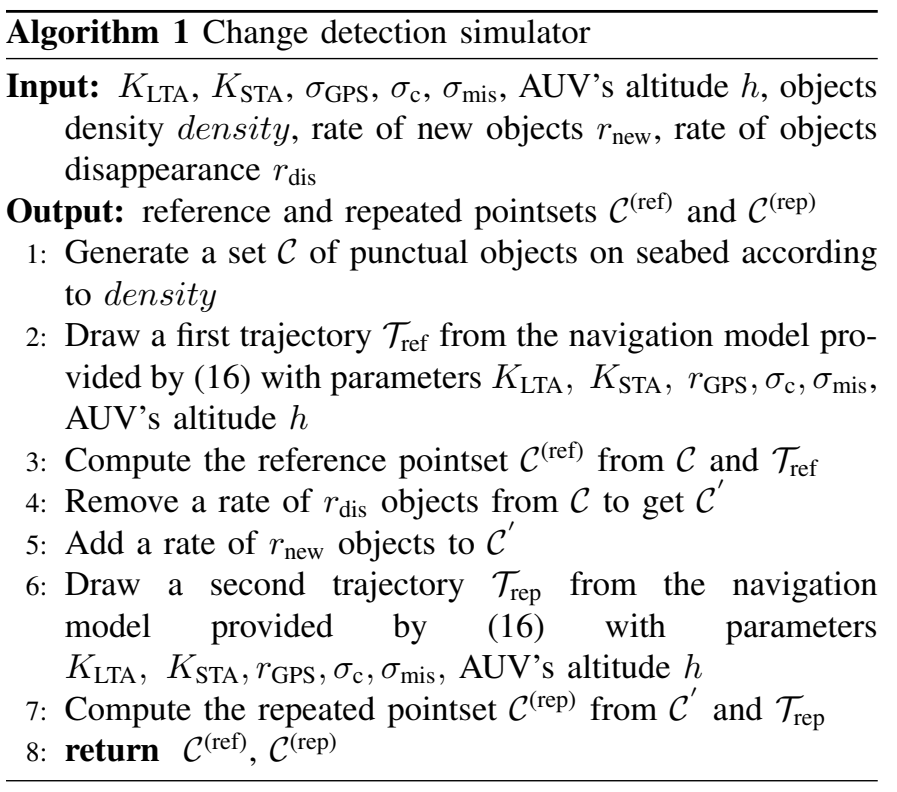

An example of trajectories $\mathcal{T}_{\text {ref }}$ and $\mathcal{T}_{\text {ref }}$ are illustrated in (Fig.4). A whole reference pointset $\mathcal{C}^{\text {(ref) }}$ is also presented in (Fig.5) while a snippet of an area after the computation of both $\mathcal{C}^{(\text {ref })}$ and $\mathcal{C}^{(\text {rep) }}$ pointsets is illustrated in (Fig.6).

\section{ICP-BASED SIMULTANEOUS REGISTRATION AND CHANGE DETECTION}

\section{A. Features extraction}

Inputs to our registration and change detection algorithm are two pointsets (17) denoted $\mathcal{C}^{(r e f)}$ and $\mathcal{C}^{(r e p)}$ respectively corresponding to the reference and the repeated ones. Each pointset gather the potentiel objects, laid on the seabed, detected in a sensed synthetic aperture side-scan sonar image. In this paper, such detections are obtained through a simple processing chain consisting of:

- SAS image denoising

- Binary image segmentation, based on the objects projected shadow, by means of hysteresis thresholding

- Mathematical morphology

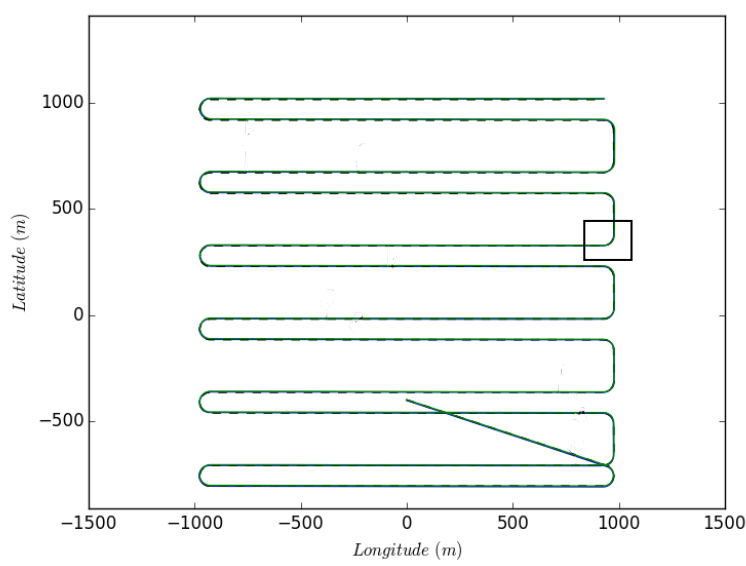

(a) $\mathcal{T}_{\text {ref }}$ and $\mathcal{T}_{\text {rep }}$ trajectories

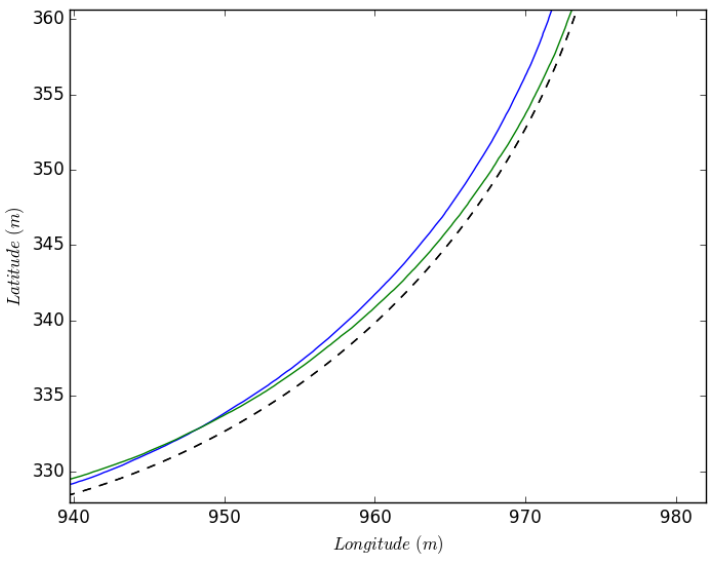

(b) Zoom on the area represented by the black box

Fig. 4: Example of our simulator output. The expected trajectory is the black dashed plot while the unobserved reference and repeated ones are respectively represented in blue and green colors.

- Objects selection based on their dimensions according to the expecting dimensions of objects of interest

These reference and repeated pointsets are mathematically described as

$$
\begin{aligned}
& \mathcal{C}^{(\text {ref })}=\left\{\left\{\mathbf{p}_{i}, \boldsymbol{\Sigma}_{P, i}\right\}\right\}_{i \in \llbracket 1, M \rrbracket} \\
& \mathcal{C}^{(r e p)}=\left\{\left\{\mathbf{q}_{j}, \boldsymbol{\Sigma}_{Q, j}\right\}\right\}_{j \in \llbracket 1, N \rrbracket},
\end{aligned}
$$

where $\boldsymbol{\Sigma}_{K, l}$ represents the covariance matrix, provided by the carrier navigation system and the ATD algorithm, associated with the detection localized at $\mathbf{k}_{l}$.

\section{B. ICP formulation}

Our simultaneous registration and change detection algorithm (Alg.2) is mainly based on the iterative closest point 


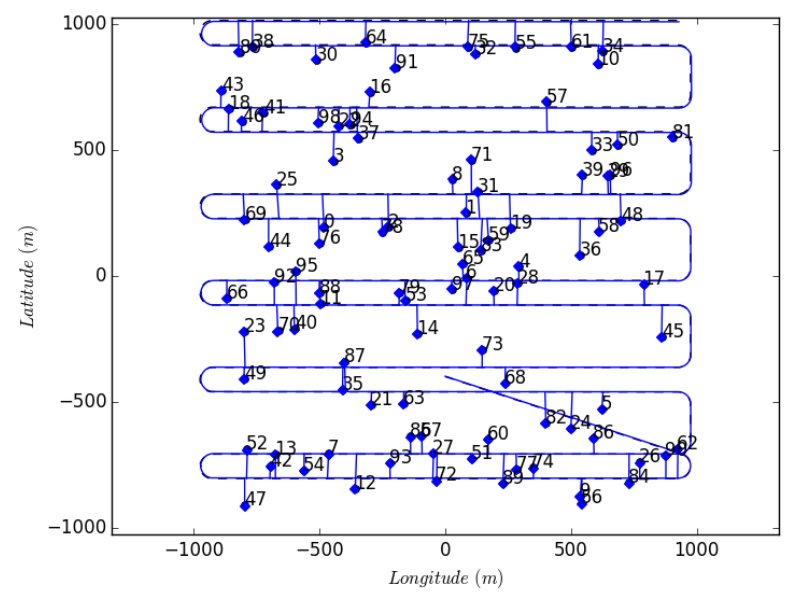

Fig. 5: A simulated reference trajectory with the computed detections.

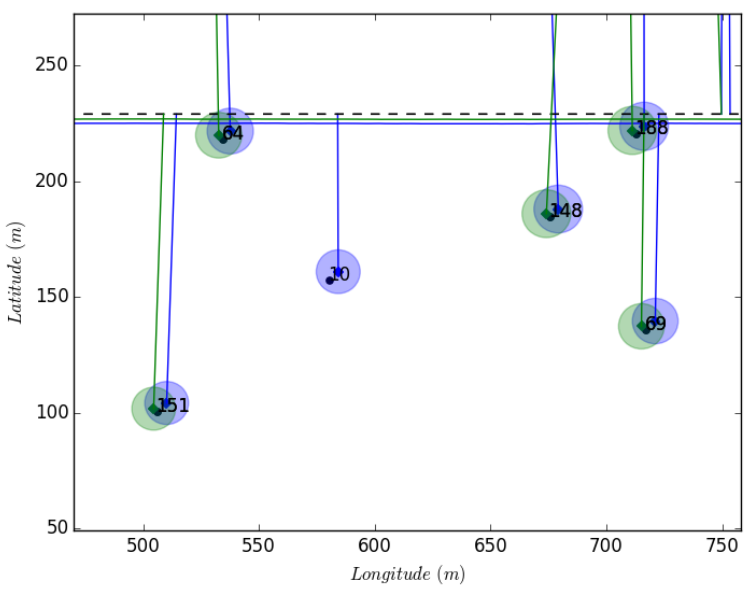

Fig. 6: Zoom on an area illustrating the true but unobserved objects locations in black while the computed reference and repeated ones are respectively represented in blue and green colors, with their respective covariance matrices.

(ICP) algorithm [33]. Indeed, the ICP algorithm iteratively alternates, until convergency, a correspondences estimation step, between points from the reference and repeated pointsets, and a pose estimation one to compute the optimal transformation. Once convergence has been achieved, a last matching and filtering step yields the final matches thus providing the objects disappeared and appeared between both tracks acquisition. The correspondences estimation step makes use of a nearest neighbors algorithm with the Mahalanobis distance.

As described by (16), the optimal transformation can roughly be characterized by a rotation matrix $\mathbf{R}^{*}$, a translation vector $\mathbf{t}^{*}$ and a scale factor $s^{*}$. In other words, we aim at computing the triplet $\mathbf{T}^{*}=\left(\mathbf{R}^{*}, \mathbf{t}^{*}, s^{*}\right)$ optimally aligning the repeated pointset $\mathcal{C}^{(r e p)}$ onto the reference one $\mathcal{C}^{(r e f)}$. $\overline{\text { Algorithm } 2 \text { Simultaneous registration and change detection }}$ algorithm

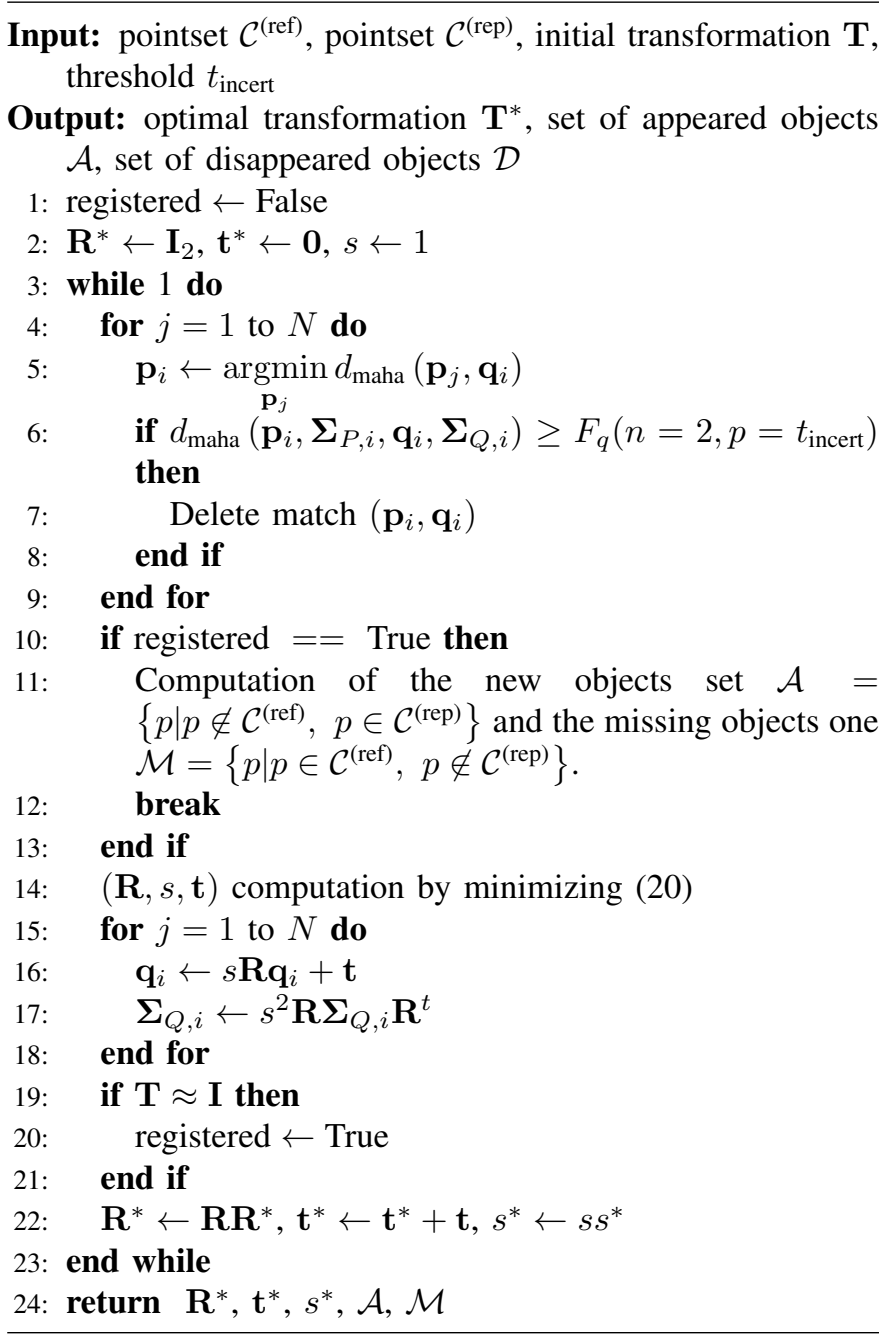

To compute these parameters, we rely on the well-known Iterative Closest Point (ICP) framework and especially on its probabilistic variant [34] to take into account the available covariance matrices.

To derive our objective function, we introduce the following residual variable $\mathbf{d}_{i}^{(\mathbf{T})}$ so that

$$
\begin{aligned}
\mathbf{d}_{i}^{(\mathbf{T})} & =\mathbf{p}_{i}-\mathbf{T} \mathbf{q}_{i} \\
& \sim \mathcal{N}\left(\overline{\mathbf{p}}_{i}-\mathbf{T} \overline{\mathbf{q}}_{i}, \boldsymbol{\Sigma}_{P, i}+\mathbf{T} \boldsymbol{\Sigma}_{i}^{(Q)} \mathbf{T}^{t}\right)
\end{aligned}
$$

The maximum likelihood estimator, under the observations independence assumption, can thus be approximated [35] by 


$$
\begin{aligned}
\mathbf{T}^{*} & =\underset{\mathbf{T}}{\operatorname{argmax}} \prod_{i} p\left(\mathbf{d}_{i}^{(\mathbf{T})}\right) \\
& =\underset{\mathbf{T}}{\operatorname{argmin}} \sum_{i} \log \operatorname{det} \boldsymbol{\Sigma}_{P, i}+\mathbf{T} \boldsymbol{\Sigma}_{Q, i} \mathbf{T}^{t}+ \\
& \mathbf{d}_{i}^{(\mathbf{T})^{t}}\left(\boldsymbol{\Sigma}_{P, i}+\mathbf{T} \boldsymbol{\Sigma}_{Q, i} \mathbf{T}^{t}\right)^{-1} \mathbf{d}_{i}^{(\mathbf{T})} \\
& \approx \underset{\mathbf{T}}{\operatorname{argmin}} \sum_{i} \mathbf{d}_{i}^{(\mathbf{T})^{t}}\left(\boldsymbol{\Sigma}_{P, i}+\mathbf{T} \boldsymbol{\Sigma}_{Q, i} \mathbf{T}^{t}\right)^{-1} \mathbf{d}_{i}^{(\mathbf{T})} .
\end{aligned}
$$

Thus, in this paper, (19) is explicitly expressed as

$$
\begin{aligned}
\mathbf{R}^{*}, \mathbf{t}^{*}, s^{*}=\underset{\mathbf{R}, s, \mathbf{t}}{\operatorname{argmin}} \sum_{\left(\mathbf{p}_{i}, \mathbf{q}_{i}\right) \in \mathcal{M}}\left(\mathbf{p}_{i}-s \mathbf{R} \mathbf{q}_{i}-\mathbf{t}\right)^{t} \\
\\
\left(\boldsymbol{\Sigma}_{P, i}+s^{2} \mathbf{R} \boldsymbol{\Sigma}_{Q, i} \mathbf{R}^{t}\right)^{-1}\left(\mathbf{p}_{i}-s \mathbf{R} \mathbf{q}_{i}-\mathbf{t}\right) .
\end{aligned}
$$

While a least-squared closed-form solution exists in the standard ICP formulation [36], [37] and in the case of isotropic covariance matrices, (20) is usually solved through iterative procedures [35], [38], [39]. In this paper, we numerically solved (20) by means of conjugate gradient descent as mentioned in the original generalized ICP paper [34]. Indeed, we initially implemented both [38] and [35] and found out that they perform identically on datasets output by the previously detailed simulator. Gradients are also numerically computed using a finite difference method.

\section{RESUlts}

\section{A. Simulated data}

To assess the algorithm previously described in terms of registration and change detection performances, we rely on the Monte-Carlo method. Indeed, from a given set of parameters, corresponding to the inputs to (Alg.1), we draw several pairs of pointsets $\mathcal{C}^{(r e f)}$ and $\mathcal{C}^{(r e p)}$ and, as the ground truth is perfectly known, it allows us to compute various statistics such as

- PR (Pairing Rate)

- CMR (Correct Matches Rate)

- EMR (Erroneous Matches Rate)

- MDR (Rate of Missing objects correctly Detected as missing)

- NDR (Rate of New objects correctly Detected as new), computed as

$$
\begin{aligned}
& \mathrm{PR}=\frac{\text { number of correct matches }}{\text { number of expected correct matches }} \\
& \mathrm{CMR}=\frac{\text { number of correct matches }}{\text { number of correct matches }+ \text { number of erroneous matches }} \\
& \mathrm{MDR}=\frac{\text { number of erroneous matches }}{\text { number of correct matches }+ \text { number of erroneous matches }} \\
& \mathrm{NDR}=\frac{\text { number of objects detected as missing and really missing }}{\text { total number of really missing objects }} \\
& \text { total number of really new objects }
\end{aligned}
$$

We have thus evaluated the robustness of our algorithm against different parameters as illustrated in (Fig.7). The results particularly show that our algorithm is fairly robust to parameters related to the navigation model while being more sensitive to new and missing objects rates.

\section{B. Evaluation on real data}

The proposed algorithm has also been evaluated on real data. Indeed, we considered two datasets, described below.

1) Homogeneous seabed: The first pair is made of two high resolution synthetic aperture sonar tracks acquired with the THALES SAMDIS sensor, mounted on an AUV equipped with a Teledyne Workhorse DVL and a iXBlue PHINS INS. These tracks have been sensed three months apart on an homogeneous seabed, mainly consisting in few mine-like objects. As some of these objects were added or removed between both tracks acquisition, it remains a challenge. The overlapping area is divided into two sub-areas namely the south and north ones (Fig.10 (a), (b))).

As the ground truth was available for this dataset, change detection performances can be assessed. These registration and change detection results are provided in (Fig.10 (c), (d)). On this dataset, the proposed algorithm has been able to detect all the new and missing objects except one missing object (not visible in (Fig.10)). Regarding the registration result, it visually shows the ability of the algorithm to match the corresponding detections to visually align corresponding features.

2) Complex seabed: This second pair is much more challenging. Indeed, not only these tracks have been sensed by means of the THALES T-SAS TSM 2054 NG (sold to the French Navy under the name DUBM-44), consisting in a SAS sensor mounted on a towed fish, but the repeated track has been acquired with a strongly rough sea state (estimated from 4 to 5 on the Douglas scale), thus causing the expected relative geometric transformation to be strongly non rigid. Moreover, the seabed can be described as complex as it is heavily cluttered while rocky structures and sand ripples also appear. To exploit this data using our algorithm, we only considered part of the data where the deformation model can locally be modeled as a rotation and a scale. However, as no ground truth was available for this dataset for the change detection task, only the registration results are of interest.

The results are illustrated in (Fig.11), where the corresponding features such as rocky structures or mine-like objects are correctly matched.

A quantitative comparison, regarding the registration performances only, has also been done between the proposed algorithm and the one described in [2] which is based on a multi-resolution correlation-based block-matching approach.

To do so, for both pairs, a ground-truth vector field has been manually retrieved in order to evaluate the different methods. Both the ground-truth vector field and the estimated one are firstly interpolated. According to a regular grid, we thus 


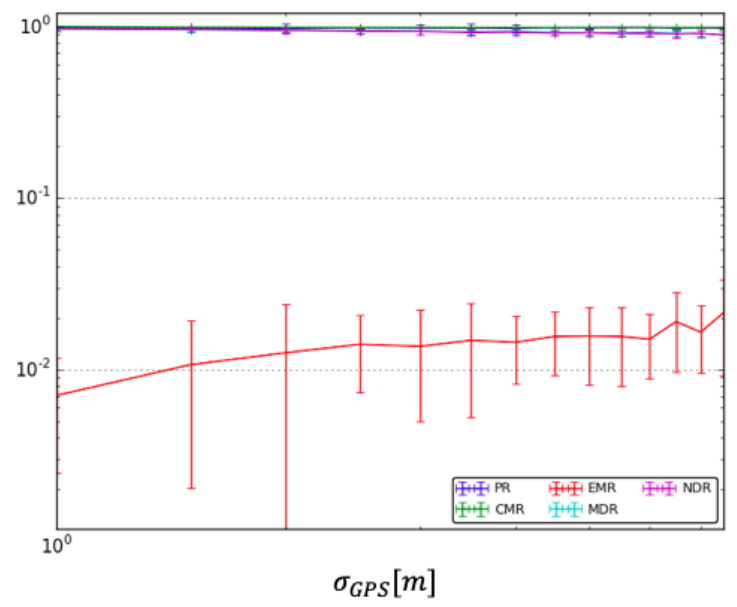

(a) Metrics with respect to $\sigma_{\mathrm{GPS}}$



(c) Metrics with respect to objects density

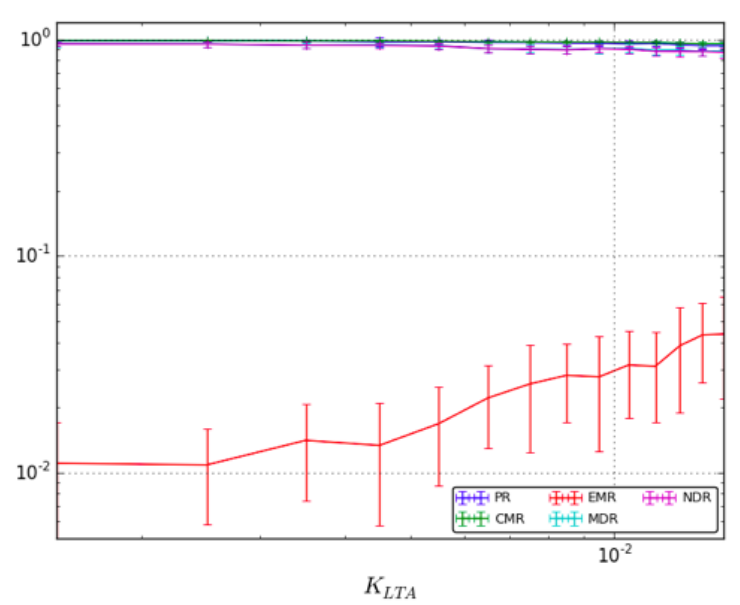

(b) Metrics with respect to $K_{\mathrm{LTA}}$

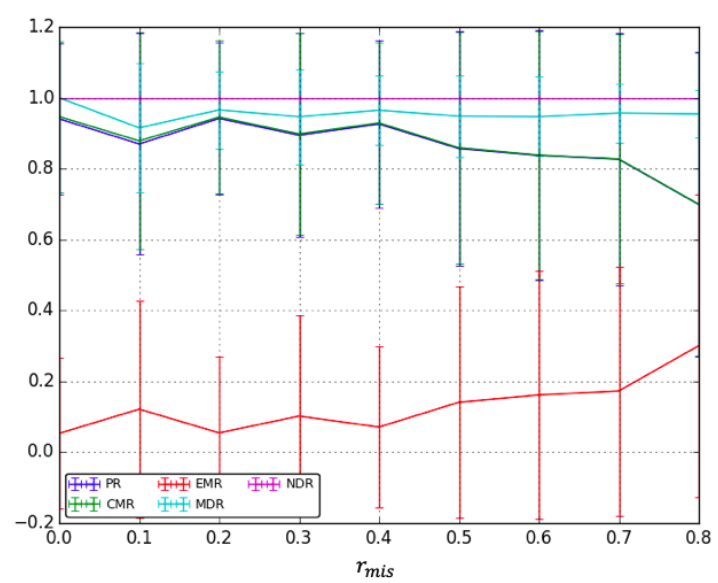

(d) Metrics with respect to the fraction of missing objects

Fig. 7: Results of the Monte-Carlo method to assess the robustness of our algorithm against various input parameters. The fixed parameters are $K_{\mathrm{LTA}}=1.5 \times 10^{-3}, K_{\mathrm{STA}}=3.0 \times 10^{-2}, \sigma_{\mathrm{GPS}}=2 \mathrm{~m}, \sigma_{\mathrm{mis}}=1^{\circ}, \sigma_{\mathrm{c}}=20 \mathrm{~m} . \mathrm{s}^{-1}, r_{\text {new }}=0.1, r_{\mathrm{mis}}=0.1$.

sample pairs of ground-truth and estimated vectors $\left\{\mathbf{v}_{i}, \mathbf{w}_{i}\right\}$, at the same locations, to respectively build the sets $\mathcal{V}$ and $\mathcal{W}$.

The designed metric $S$ is then computed as

$$
S=D_{d}(\mathcal{V}, \mathcal{W})+\lambda D_{a}(\mathcal{V}, \mathcal{W})
$$

where $\lambda$ has been set to 0.1 and

$$
\begin{aligned}
& D_{d}(\mathcal{V}, \mathcal{W})=\frac{1}{\operatorname{card}[\mathcal{V}]} \sum_{\left(\mathbf{v}_{i}, \mathbf{w}_{i}\right) \in \mathcal{V} \times \mathcal{W}}\left\|\mathbf{v}_{i}-\mathbf{w}_{i}\right\| \\
& D_{a}(\mathcal{V}, \mathcal{W})=\frac{1}{\operatorname{card}[\mathcal{V}]} \sum_{\left(\mathbf{v}_{i}, \mathbf{w}_{i}\right) \in \mathcal{V} \times \mathcal{W}} \mathbf{v}_{i} \ominus \mathbf{w}_{i},
\end{aligned}
$$

where $\ominus$ stands for the angular difference between two vectors.

Numerical results are provided in (Tab.I) and demonstrate that the vector field yielded by the proposed algorithm achieves

\begin{tabular}{|c|c|c|}
\hline Seabed type & Algo. & $S$ \\
\hline \multirow{3}{*}{ Homogeneous } & Multi-resolution correlation [2] & 1.59 \\
\cline { 2 - 3 } & M-ICP [ours] & $\mathbf{1 . 6 9}$ \\
\cline { 2 - 3 } & SIFT [40] & $\mathbf{1 . 3 7}$ \\
\cline { 2 - 3 } & SURF [41] & 1.83 \\
\cline { 2 - 3 } Complex & BRISK [42] & 2.47 \\
\cline { 2 - 3 } & Multi-resolution correlation [2] & $\mathbf{0 . 5 5}$ \\
\cline { 2 - 3 } & M-ICP [ours] & $\mathbf{0 . 9 0}$ \\
\cline { 2 - 3 } & SIFT [40] & 1.24 \\
\cline { 2 - 3 } & SURF [41] & 2.02 \\
\cline { 2 - 3 } & BRISK [42] & 1.73 \\
\hline
\end{tabular}

TABLE I: Quantitative comparison between different registration algorithms

slightly worse results than the multi-resolution intensity-based approach on both datasets while still being superior to common keypoints detectors, except for the SIFT algorithm which performs better on the homogeneous dataset. 


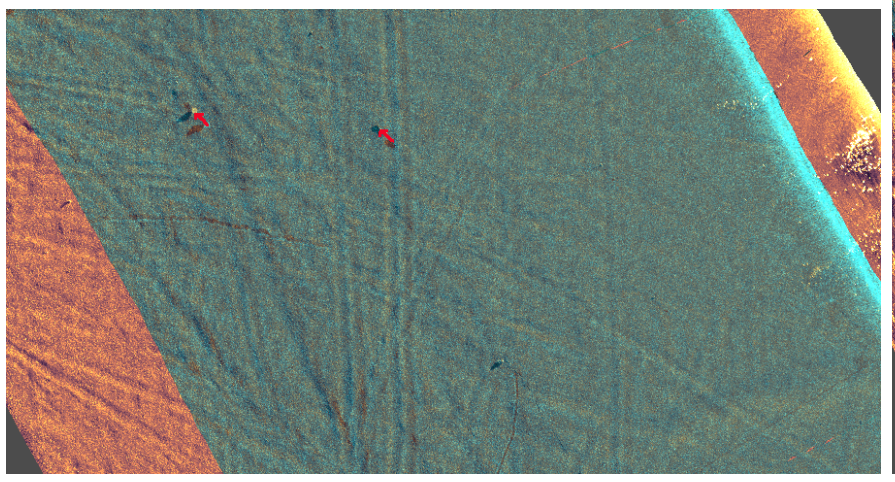

(a) North area before registration

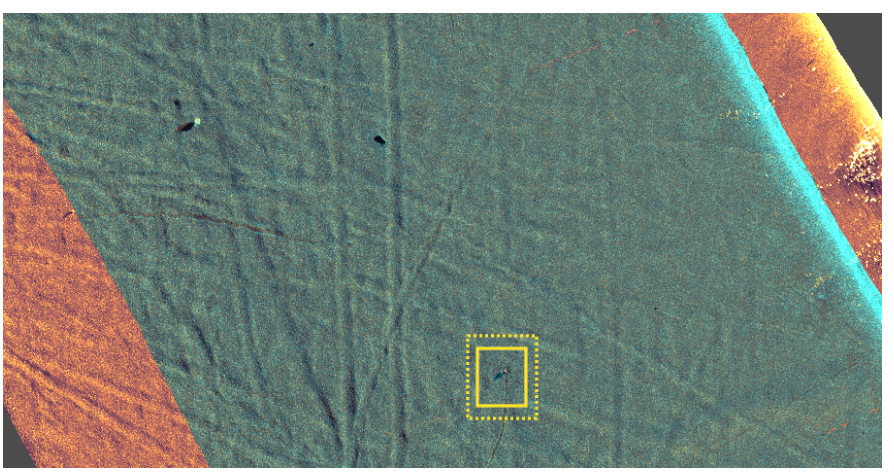

(c) North area after registration



(b) South area before registration

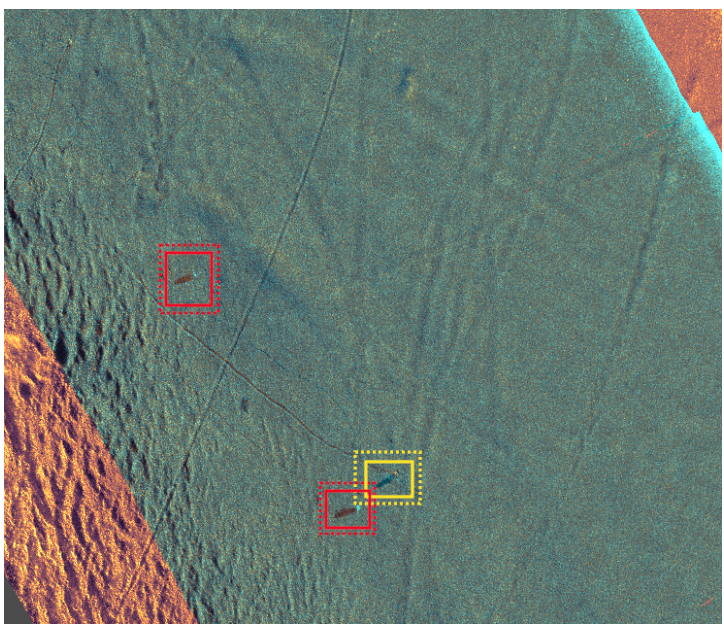

(d) South area after registration

Fig. 8: Registration and change detection results for the homogeneous seabed dataset. The estimated vector field is denoted by red arrows. Boxes illustrate the change detection result. Solid yellow line boxes correspond to objects detected as being new while dashed line ones are the real new objects according to the ground truth. Solid red line boxes correspond to objects detected as missing while dashed line ones are the real missing objects according to the ground truth.

\section{CONCLUSION AND FURTHER WORK}

In this paper, we proposed a symbolic simultaneous registration and change detection algorithm, strongly based on the probabilistic iterative closest point formulation, in the underwater mine warfare context. To assess the robustness of such an algorithm we have designed a simulator whose the main component is a navigation model based on sensors the carrier is equipped with. This algorithm has then been experimented on datasets acquired at sea on two different seabed types namely a homogenous and a complex ones, thus demonstrating its interest to solve the change detection problem. Compared to purely image-based methods, change detection performances are usually better for a symbolic approach as several invariances (grazing and view angles) are taken into account during the features extraction step. However, in case of missing or new objects, the robustness of the matching step can be poor, compared to image-based methods that can benefit from multifarious common structures present in both datasets.

Further work would thus consists in modifying the distance used within the nearest neighbors matching step, in order to incorporate more than just a geometric distance. Indeed, a greater robustness could potentially be achieved by adding other descriptors such as shape contexts [43] or even image- 


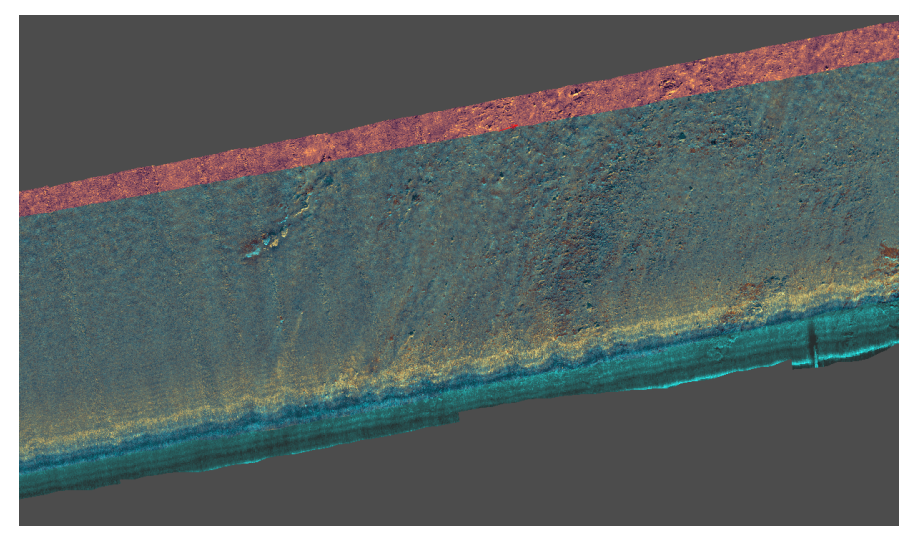

(a) West area before registration

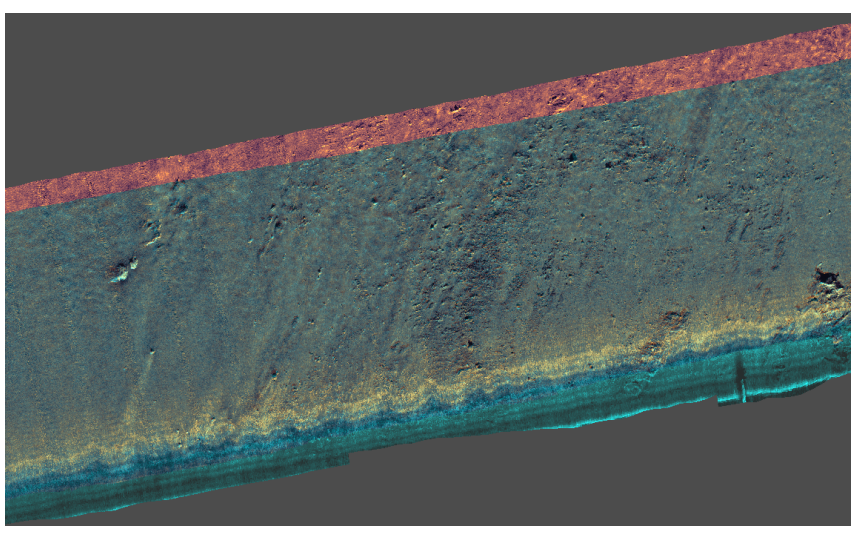

(b) West area after registration

Fig. 9: Registration results for the western area of the complex seabed dataset.

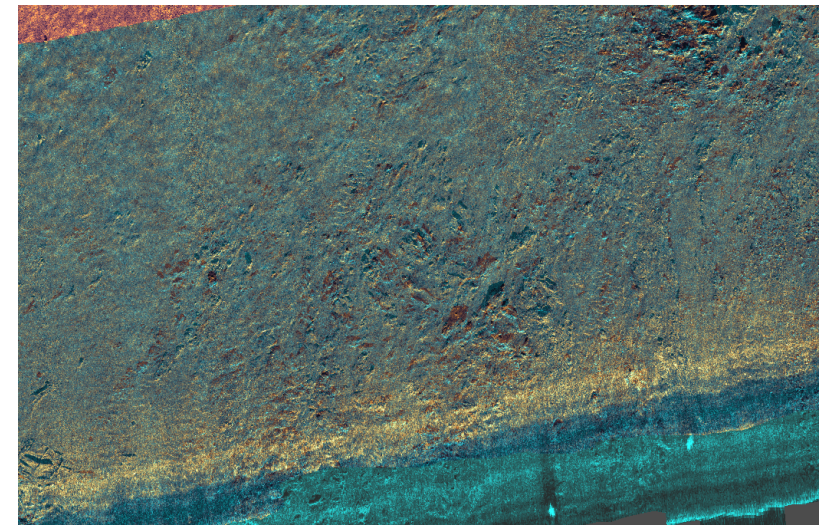

(a) Center area before registration

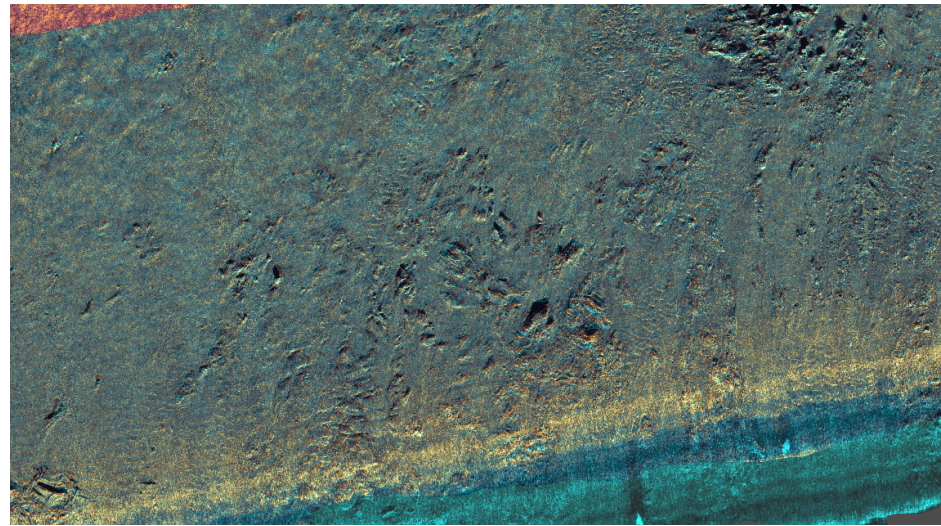

(b) Center area after registration

Fig. 10: Registration results for the centered area of the complex seabed dataset.

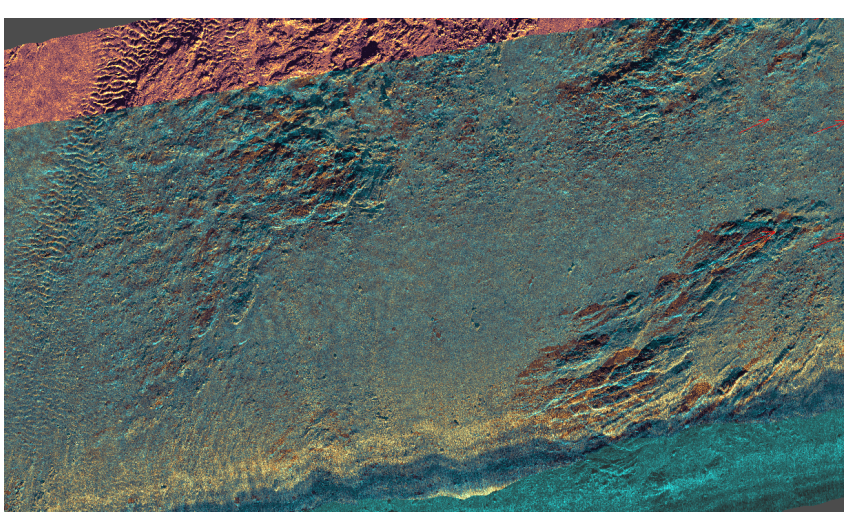

(a) East area before registration

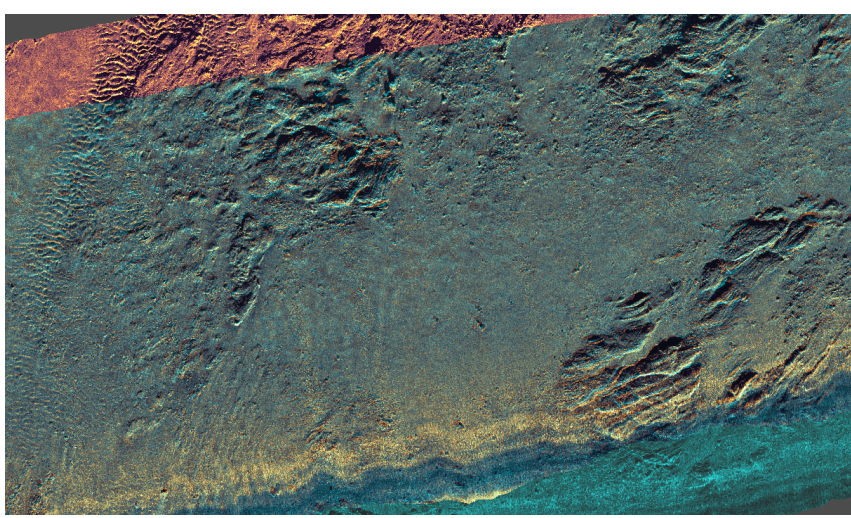

(b) East area after registration

Fig. 11: Registration results for the eastern area of the complex seabed dataset 
based descriptors [40].

\section{ACKNOWLEDGMENTS}

The authors would like to thank the Direction Générale de l'Armement (DGA) for their financial support and for providing both the SAMDIS and T-SAS datasets.

\section{REFERENCES}

[1] C. Chailloux, J.-M. Le Caillec, D. Gueriot, and B. Zerr, "Intensity-based block matching algorithm for mosaicing sonar images," IEEE Journal of Oceanic Engineering, vol. 36, no. 4, pp. 627-645, 2011.

[2] F. Nicolas, A. Arnold-Bos, I. Quidu, and B. Zerr, "Fourier-based blockmatching algorithm for non-rigid sonar images registration in a multiresolution framework," MOQESM'16, 2016.

[3] P.-Y. Mignotte, M. Lianantonakis, and Y. Petillot, "Unsupervised registration of textured images: applications to side-scan sonar," in Oceans 2005-Europe, vol. 1. IEEE, 2005, pp. 622-627.

[4] N. Hurtós, Y. Petillot, J. Salvi et al., "Fourier-based registrations for two-dimensional forward-looking sonar image mosaicing," in Intelligent Robots and Systems (IROS), 2012 IEEE/RSJ International Conference on. IEEE, 2012, pp. 5298-5305.

[5] S. Dugelay and W. Fox, "Active contours for synthetic aperture sonar snippet registration," in OCEANS 2015-Genova. IEEE, 2015, pp. 1-6.

[6] F. Nicolas, A. Arnold-Bos, I. Quidu, and B. Zerr, "Markov-based approaches for ternary change detection between two high resolution synthetic aperture sonar tracks," in OCEANS 2017 - Aberdeen, June 2017, pp. 1-9.

[7] Ø. Midtgaard, R. E. Hansen, T. O. Sæbø, V. Myers, J. R. Dubberley, and I. Quidu, "Change detection using synthetic aperture sonar: Preliminary results from the Larvik trial," in OCEANS 2011. IEEE, 2011, pp. 1-8.

[8] D. Guériot, E. Maillard, and J.-P. Kernin, "Sonar image registration through symbolic matching: A fuzzy local transform approach using genetic algorithms," in OCEANS'96. MTS/IEEE. Prospects for the 21 st Century. Conference Proceedings, vol. 3. IEEE, 1996, pp. 1324-1329.

[9] S. Daniel, F. Le Leannec, C. Roux, B. Soliman, and E. P. Maillard, "Side-scan sonar image matching," IEEE Journal of Oceanic Engineering, vol. 23, no. 3, pp. 245-259, 1998.

[10] I. Leblond, M. Legris, and B. Solaiman, "Use of classification and segmentation of sidescan sonar images for long term registration," in Oceans 2005-Europe, vol. 1. IEEE, 2005, pp. 322-327.

[11] I. Barrodale, R. Kuwahara, R. Poeckert, and D. Skea, "Side-scan sonar image processing using thin plate splines and control point matching," Numerical Algorithms, vol. 5, no. 2, pp. 85-98, 1993.

[12] J. Ferrand and N. Mandelert, "Change detection for MCM survey mission," in 2012 International Conference on Detection and Classification of Underwater Targets, 2012, pp. 193-206.

[13] B. Bonnett and M. Hayes, "Data-driven image registration for coherent change detection of synthetic aperture sonar imagery," in Proceedings of the 29th International Conference on Image and Vision Computing New Zealand. ACM, 2014, pp. 196-201.

[14] J. Dillon and V. Myers, "Coherence estimation for repeat-pass interferometry," in Oceans-St. John's, 2014. IEEE, 2014, pp. 1-5.

[15] G. Tesfaye, B. Marchand, J. D. Tucker, T. M. Marston, D. D. Sternlicht, M. R. Azimi-Sadjadi et al., "Image-based automated change detection for synthetic aperture sonar by multistage coregistration and canonical correlation analysis," IEEE Journal of Oceanic Engineering, vol. 41, no. 3, pp. 592-612, 2016

[16] I. Quidu, V. Myers, Ø. Midtgaard, and R. E. Hansen, "Subpixel image registration for coherent change detection between two high resolution sonar passes," in ICoURS'12, 2012.

[17] D. D. Sternlicht, J. K. Harbaugh, and M. A. Nelson, "Experiments in coherent change detection for synthetic aperture sonar," in OCEANS 2009, MTS/IEEE Biloxi-Marine Technology for Our Future: Global and Local Challenges. IEEE, 2009, pp. 1-5.

[18] T. O. Sæbø, R. E. Hansen, H. J. Callow, and S. Synnes, "Coregistration of synthetic aperture sonar images from repeated passes," Proceedings of Underwater Acoustic Measurements, 2011.

[19] A. P. Lyons and D. C. Brown, "The impact of the temporal variability of seafloor roughness on synthetic aperture sonar repeat-pass interferometry," IEEE Journal of Oceanic Engineering, vol. 38, no. 1, pp. 91-97, 2013.
[20] B. Jalving, E. Bovio, and K. Gade, "Integrated inertial navigation systems for AUVs for REA applications," in SACLANTCEN conference proceedings from MREP, 2003.

[21] I. T. Ruiz, S. De Raucourt, Y. Petillot, and D. M. Lane, "Concurrent mapping and localization using sidescan sonar," IEEE Journal of Oceanic Engineering, vol. 29, no. 2, pp. 442-456, 2004.

[22] M. F. Fallon, M. Kaess, H. Johannsson, and J. J. Leonard, "Efficient AUV navigation fusing acoustic ranging and side-scan sonar," in Robotics and Automation (ICRA), 2011 IEEE International Conference on. IEEE, 2011, pp. 2398-2405.

[23] L. Bernicola, D. Guériot, and J.-M. L. Caillec, "A hybrid registration approach combining slam and elastic matching for automatic side-scan sonar mosaic," 2014 Oceans - St. John's, pp. 1-5, 2014.

[24] T. M. Joyce, "On in situ calibration of shipboard ADCPs," Journal of Atmospheric and Oceanic Technology, vol. 6, no. 1, pp. 169-172, 1989.

[25] B. Jalving, K. Gade, K. Svartveit, A. Willumsen, and R. Sørhagen, "DVL velocity aiding in the Hugin 1000 integrated inertial navigation system," Modeling, Identification and Control, vol. 25, no. 4, p. 223, 2004.

[26] Y. Doisy, "Theoretical accuracy of doppler navigation sonars and acoustic doppler current profilers," IEEE Journal of Oceanic Engineering, vol. 29, no. 2, pp. 430-441, 2004

[27] O. J. Woodman, "An introduction to inertial navigation," University of Cambridge, Computer Laboratory, Tech. Rep., 2007.

[28] J. Rankin, "An error model for sensor simulation GPS and differential GPS," in Position Location and Navigation Symposium, 1994., IEEE, Apr 1994, pp. 260-266

[29] I. Quidu, N. Burlet, J.-P. Malkasse, and F. Florin, "Automatic classification for MCM systems," in Oceans 2005-Europe, vol. 2. IEEE, 2005, pp. 844-847.

[30] J. McKay, I. Gerg, V. Monga, and R. Raj, "What's mine is yours: Pretrained CNNs for limited training sonar ATR," arXiv preprint arXiv:1706.09858, 2017.

[31] D. P. Williams, "Underwater target classification in synthetic aperture sonar imagery using deep convolutional neural networks," in Pattern Recognition (ICPR), 2016 23rd International Conference on. IEEE, 2016, pp. 2497-2502.

[32] V. Myers and J. Fawcett, "A template matching procedure for automatic target recognition in synthetic aperture sonar imagery," IEEE Signal Processing Letters, vol. 17, no. 7, pp. 683-686, 2010.

[33] P. J. Besl, N. D. McKay et al., "A method for registration of 3-d shapes," IEEE Transactions on pattern analysis and machine intelligence, vol. 14, no. 2, pp. 239-256, 1992.

[34] A. Segal, D. Haehnel, and S. Thrun, "Generalized-ICP." in Robotics: science and systems, vol. 2, no. 4, 2009, p. 435.

[35] S. D. Billings, E. M. Boctor, and R. H. Taylor, "Iterative most-likely point registration (IMLP): a robust algorithm for computing optimal shape alignment," PloS one, vol. 10, no. 3, p. e0117688, 2015.

[36] K. S. Arun, T. S. Huang, and S. D. Blostein, "Least-squares fitting of two 3-d point sets," IEEE Transactions on pattern analysis and machine intelligence, no. 5, pp. 698-700, 1987.

[37] T. Zinßer, J. Schmidt, and H. Niemann, "Point set registration with integrated scale estimation," in International Conference on Pattern Recognition and Image Processing, 2005, pp. 116-119.

[38] R. S. J. Estépar, A. Brun, and C.-F. Westin, "Robust generalized total least squares iterative closest point registration," in International Conference on Medical Image Computing and Computer-Assisted Intervention. Springer, 2004, pp. 234-241.

[39] L. Maier-Hein, A. M. Franz, T. R. dos Santos, M. Schmidt, M. Fangerau, H.-P. Meinzer, and J. M. Fitzpatrick, "Convergent iterative closest-point algorithm to accomodate anisotropic and inhomogenous localization error," IEEE transactions on pattern analysis and machine intelligence, vol. 34 , no. 8 , pp. 1520-1532, 2012.

[40] D. G. Lowe, "Distinctive image features from scale-invariant keypoints," International journal of computer vision, vol. 60, no. 2, pp. 91-110, 2004.

[41] H. Bay, T. Tuytelaars, and L. Van Gool, "Surf: Speeded up robust features," Computer vision-ECCV 2006, pp. 404-417, 2006.

[42] S. Leutenegger, M. Chli, and R. Y. Siegwart, "BRISK: Binary robust invariant scalable keypoints," in Computer Vision (ICCV), 2011 IEEE International Conference on. IEEE, 2011, pp. 2548-2555.

[43] S. Belongie, J. Malik, and J. Puzicha, "Shape context: A new descriptor for shape matching and object recognition," in Advances in neural information processing systems, 2001, pp. 831-837. 